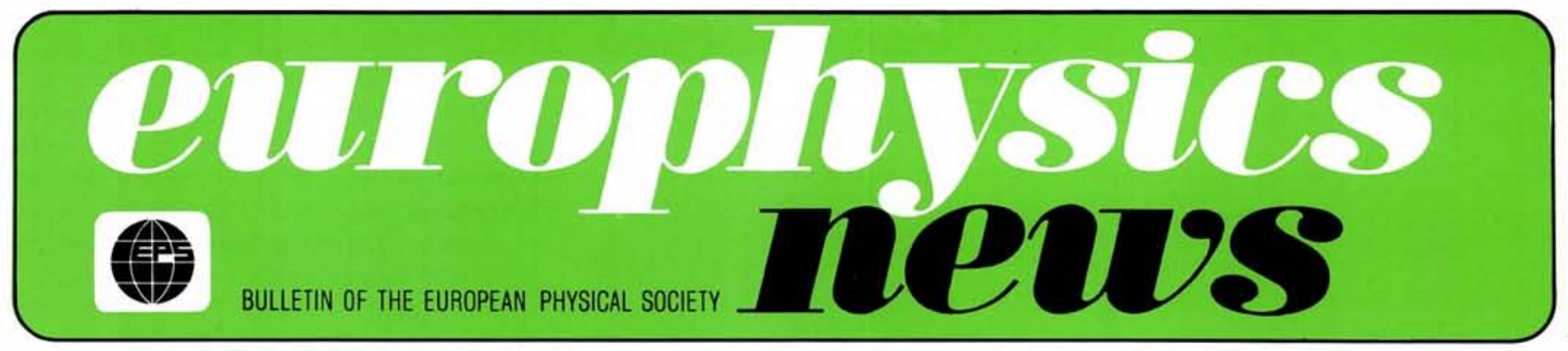

J.A.

\title{
Molecular Beam Studies of Elementary Photophysical Processes
}

\author{
Ingolf Volker Hertel, Berlin
}

(Institut für Molekülphysik der Freien Universität Berlin)

\section{Electronic energy and charge transfer, model cases on the way towards an ab initio understanding of photophysics and-chemistry.}

Photophysical and photochemical processes are of fundamental importance in a multitude of applications in physics, technology, chemistry and biology and examples may be cited as varied as materials processing with lasers, optical desorption from the surfaces of solids, photolysis of organic molecules and primary mechanisms of photosynthesis by plants. All of these have one feature in common: the modification or initiation of the molecular dynamics by light, although in general, the underlying mechanisms are extremely complex and involve an interwoven chain of many different elementary steps leading from the primary absorption of one or several photons to the final products.

From a physicist's point of view a number of exciting questions thus arise: Where in the system is the initial photon energy deposited? To where and how is this energy transferred and subsequently converted? Are photons re-emitted? Are free charges generated? Are electrons or electron-hole pairs exchanged between individual atoms and molecules? Which are the preferred pathways and reactants? What intermediate fragments or radicals are involved? What are the characteristic time scales for individual steps? And so on. A substantial fraction of the effort in present day atomic and molecular physics is devoted to a detailed understanding of such questions.

What is considered a detailed understanding depends of course very much on the personal viewpoint of the scientist studying these phenomena. Only in very rare cases can one match U. Fano's ultimate standards which he formulated as follows: "To my mind the ultimate goal of all low-energy physics is the elucidation of physico-chemical elementary processes in wavemechanical terms". Normally one will have to be content with much less. For this reason it is all the more important to develop simple models of the fundamental processes and test them in a set of sufficiently simple cases against $a b$ initio calculations, i.e. calculations based on Schrödinger's equation. Thus, the molecular physicist is concerned with the individual steps of the complex processes mentioned above and tries to isolate them from each other. The ultimate approach is to study only two individual atoms or molecules at a time, expose one of them to photons of well-defined frequency, intensity and time structure and see what happens. If possible one also wants to determine the internal state of the reactants prior to the interaction as well as their relative kinetic energy. Alternatively or, even better in addition, one likes to detect all the product particles, their internal state distribution and their translational momenta, thus tracing back what has happened during the entire process. Finally, one wants to compare the outcome of such an experiment with theoretical analyses based on quantum mechanics.

A schematic of such a photophysical experiment with complete 'state selec- tivity' is depicted in Fig. 1. In its general form such an 'ideal experiment' is of course an impossible monster, but based on new experimental and theoretical techniques, tremendous advances in this direction have been made in recent years. The key tools are supersonic beams producing cold atoms and molecules with well-defined velocities and narrow internal state distributions, tuneable high resolution lasers in the visible, near infrared and UV spectral regions, intense sources of synchrotron radiation in the VUV and $\mathrm{X}$-ray regions, and the coincidence techniques that have been gradually introduced into standard molecular physics experiments (as used by nuclear and high energy physics for many years).

These are complemented on the theoretical side by a new generation of computers together with advanced quantum

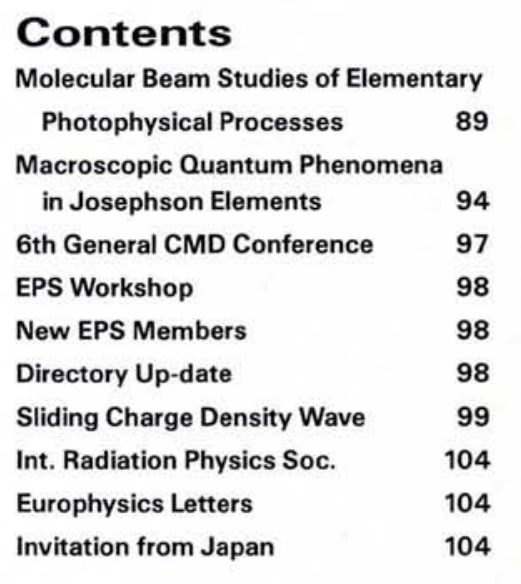




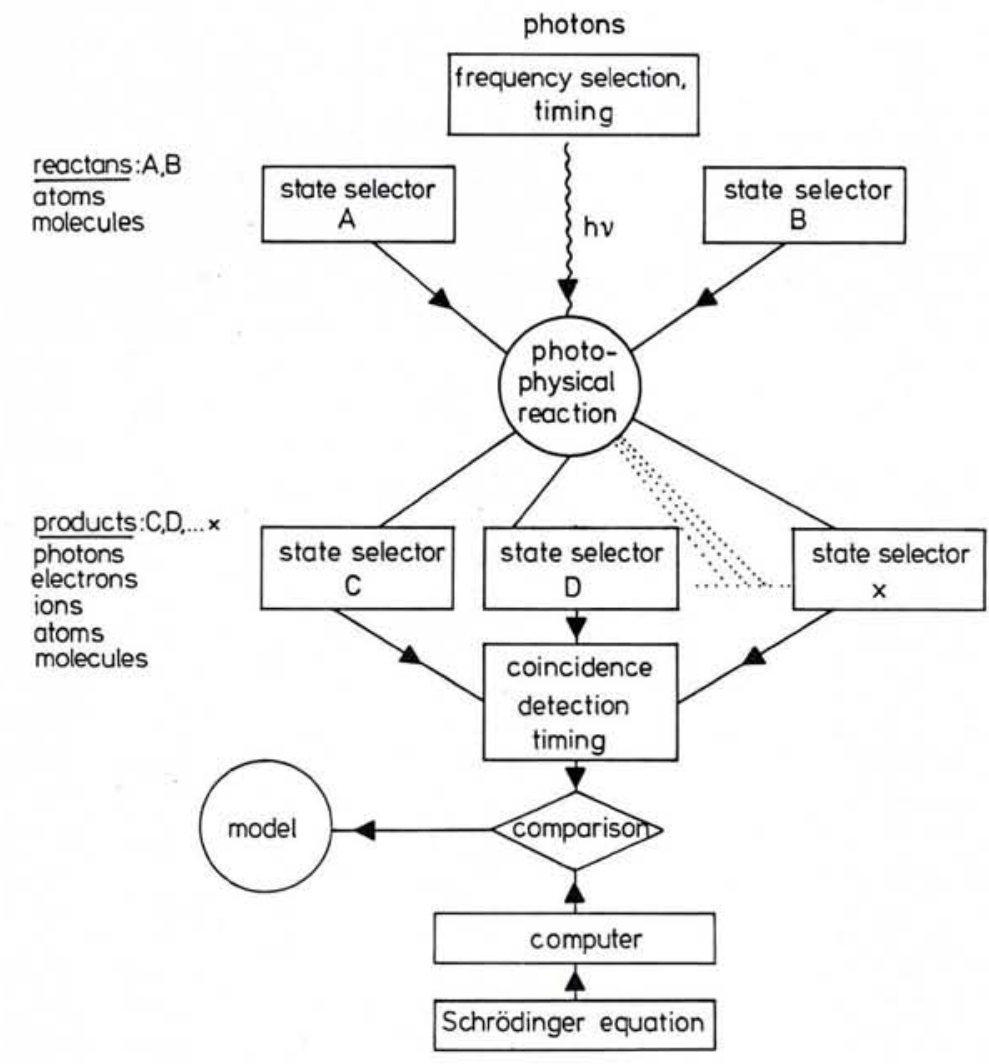

Fig. 1 -Schematic of an «ideal» photophysical experiment.

chemical methods for structure calculations and the development of powerful techniques in solving molecular dynamical and scattering problems. We should be aware, however, that in spite of the progress made, for a large number of processes involving atoms or molecules excited by photons, "the study of electronically excited states is in many respects still in its infancy" ${ }^{1}$ ) and much further study is necessary before we can dream of understanding something like, say, the primary process of charge separation in chlorophyl on this level. Here I will illustrate the present possibilities and the degree of detail that may be studied and understood by three examples of energy and charge transfer in very simple model systems, using atomic and molecular beams, lasers and synchrotron radiation. In view of the tremendous wealth of data in this field my choice of examples is extremely limited and biased by what has been studied in our own laboratory. For more examples the reader is referred to previous articles in Europhysics News ${ }^{2}$ ).

The transfer of electronic energy between an excited atom $A$ and the vibrations and rotations of a diatomic molecule BC

$$
\mathrm{A}^{*}+\mathrm{BC} \rightarrow \mathrm{A}+\mathrm{BC} \mathrm{C}^{\#}
$$

may be considered one of the most elementary precursors of a photochemical reaction. (Let ${ }^{*}$ stand for electronic and \# for rotational, vibrational excitation.)
Such processes have been studied for many years in gas cells and are known to have very large cross-sections which appear in some cases to be even larger than the gas kinetic values. In fact such studies date back to the beginning of the century when Wood ${ }^{3}$ ) discovered that the fluorescence of alkali atoms was quenched when he added foreign molecules to his atoms in a bulb illuminated by a lamp. He concluded that a "quantitative examination of this phenomenon appeared desirable". However, up until very recently the mechanism for this process was completely unclear and subject to a variety of ill-founded speculations. Since we are concerned with initial kinetic energies of the order of thermal energies (some 10 to $100 \mathrm{meV}$ ), the velocities of the interacting particles are small and the resulting electric fields change so slowly that a direct field-
Fig. 2-Experimental set up for the study of electronic to vibrational-rotational energy transfer between laser excited $\mathrm{Na}^{\circ}$ atoms and $\mathrm{H}_{2}$ molecules. induced excitation is not possible. How then is the energy from the fast electron motion in the atom transferred into the much slower motion of the atomic constituents $A, B$ and $C$ of the interacting system? Experimentally the first question to be answered is what fraction of the available energy $E_{0}$ (i.e. the initial relative kinetic energy $E_{\mathrm{cm}}$ in the centre of mass system plus the electronic energy $E_{\text {el }}$ ) goes into internal energy $E_{\text {vibrot }}$ of the molecule and relative kinetic energy $E_{\mathrm{cm}}^{\prime}$ of the receding particles after the interaction. Of course, we want to study this process under completely isolated conditions so that we are sure that one atom $A^{*}$ interacts with only one molecule $B C$ at a time and reaches our detector undisturbed by the environment.

The answer to this challenge is an experiment with crossed beams of atoms, molecules and photons tuned to a resonance transition of the atom. As a good compromise between experimental feasibility and simplicity (with hope for an ab initio understanding) we have chosen $\mathrm{Na}$ as the atom and $\mathrm{H}_{2}$ as the molecule. Fig. 2 shows the experimental set-up ${ }^{4}$ ). The $\mathrm{Na}$ atoms may, for theoretical purposes, be considered to be very like hydrogen atoms while at the same time much simpler to prepare in a beam by just letting them emerge from a heated 'oven' through a small orifice followed by apertures to skim the beam. Excitation to the first resonant state (the $3^{2} \mathrm{P}$-state) is easily achieved by a tuneable $\mathrm{cw}$ Rhodamine 6G laser. One has to do this in the interaction zone since, during the lifetime of the excited state (approx. $10^{-8}$ s), the atoms travel less than a tenth of a millimeter. The molecular $\mathrm{H}_{2}$ beam is a so-called supersonic beam created by pushing the molecules with a high pressure (a few bar) through a small nozzle. The resulting adiabatic expansion drastically cools the molecules so that only the lowest rotational states are occupied and the molecules all have a very well defined velocity $(2500 \mathrm{~m} / \mathrm{s})$ with a narrow spread (15\% FWHM). The densities

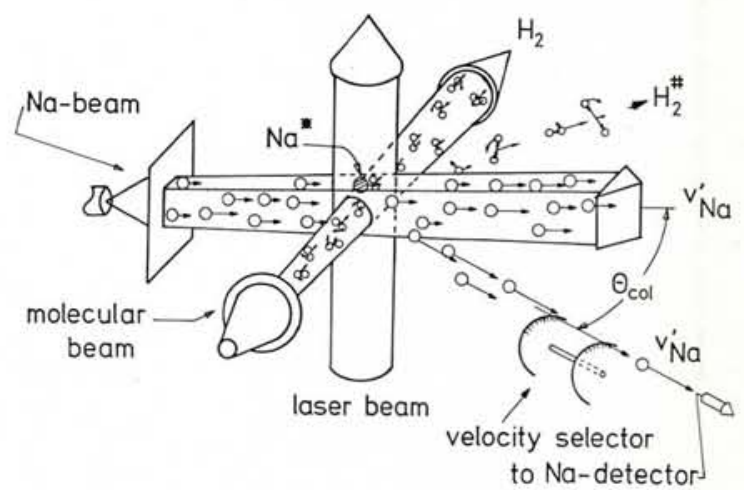




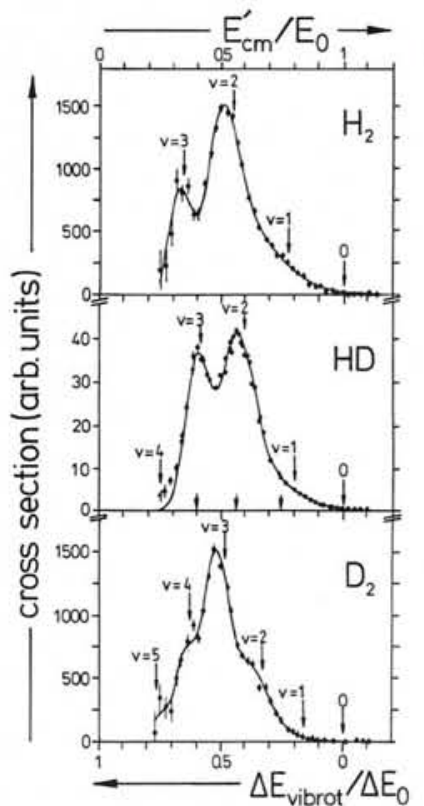

Fig. 3 - Electronic to vibrational energy transfer in the system $\mathrm{Na}^{\circ}+\mathrm{H}_{2} \rightarrow \mathrm{Na}+\mathrm{H}_{2}^{*}$ and its isotopic varieties. Experimental results from a crossed beam experiment at a total initial energy of $E_{0}=2.2 \mathrm{eV}$ and forward scattering. The final kinetic energy $\left(\mathrm{E}^{\mathrm{cm}}\right)$ of the system and the final internal energy content $\left(\mathrm{E}_{\text {vibrot }}\right)$ of the molecule are displayed in units of $E_{0}$, the internal energy in units of molecular vibrational quanta $\mathrm{v}$.

in both beams are very low $\left(10^{10}\right.$ and $10^{14} \mathrm{~cm}^{-3}$ for $\mathrm{Na}$ and $\mathrm{H}_{2}$, respectively) so that single collision conditions are guaranteed. Thus we have pretty much prepared our system in an ideal way as outlined above, the total available energy being $E_{\mathrm{o}}=E_{\mathrm{cm}}+E_{\mathrm{el}}=2.2 \mathrm{eV}$.

After the interaction process we may observe the scattered sodium atoms by ionising them on a hot wire detector and then directing them onto a particle multiplier. First however we analyse their velocity by a Fizeau type mechanical selector and by a somewhat elaborate, but standard evaluation of the experimental kinematics. We thus derive the relative kinetic energy of the system $E_{\mathrm{cm}}^{\prime}$ after the collision. The total energy balance of the process

$$
E_{0}=E_{\mathrm{cm}}+E_{\mathrm{el}}=E_{\mathrm{cm}}^{\prime}+E_{\text {vibrot }}
$$

then allows us to determine the relative amount $E_{\text {vibrot }} / E_{0}$ of internal vibrationalrotational energy transferred to the molecule in this process, the key quantity in understanding the underlying mechanisms. Fig. 3 shows the experimental results for $\mathrm{Na}^{*}+\mathrm{H}_{2}$ as well as those obtained by replacing the hydrogen molecule by its isotopic varieties $H D$ and $D_{2}$.

We clearly see that the most probable vibrational quantum $v$ of the molecule to be excited is 2 for $\mathrm{H}_{2}, 3$ for $\mathrm{D}_{2}$ while for $\mathrm{HD}, v=2$ and 3 are about equally probable. On the energy scale, however, we see that in all three cases about the same amount of energy is transferred into the molecule: The molecule accepts a rather well-defined energy rather than a well-defined number of quanta of vibration. This is a very significant finding and leads us to the key in understanding the mechanisms of the process. In all three cases the potential energy of the combined system as a function of the internuclear coordinates is identical. Thus we expect that it is the potential energy surface and the symmetry and structure of the electronic states which determine the essential features of the process. Fortunately it is possible to resort to rather reliable quantum chemical calculations for systems of this type ${ }^{5}$ ). Meanwhile a number of other systems such as $\mathrm{Na}^{*}+\mathrm{N}_{2}$ and $\mathrm{Na}^{*}+\mathrm{CO}$ have been studied experimentally and theoretically, including even dynamical calculations; see e.g. the CO case ${ }^{6}$ ). Based on these results an intuitively very convincing picture emerges which, giving the main facts in a nutshell, is schematically depicted in Fig. 4. It illustrates the charge cloud of the atomic electron (initially in a $3 p$ orbital) at consecutive moments of a typical individual collision process, i.e. as a function of the distance between the hydrogen molecule and the $\mathrm{Na}$ atom. It also illustrates the motion of the two hydrogen atoms during this interaction; for simplicity the $\mathrm{Na}$ atom is shown at rest, so the $\mathrm{H}_{2}$ trajectory represents the relative motion.

What happens is the following: As the hydrogen approaches the excited $\mathrm{Na}$ atom with its large $3 p$ electron orbital (Fig. 4a) a fraction of this electron cloud is transferred to the $\mathrm{H}_{2}$ molecule. It fills an unoccupied $\sigma_{u}$ orbital of the $\mathrm{H}_{2}$ (Fig. $4 \mathrm{~b})$, which has the same symmetry as the $3 p$ orbital for the geometry illustrated in the figure. This has two consequences: (1) it imparts a somewhat ionic character to the collision partners and thus leads to mutual attraction and (2) since the $\sigma_{u}$ orbital is antibonding, it weakens the molecular bond, i.e. it slowly increases the distance of the two hydrogen atoms as $\mathrm{H}_{2}$ and $\mathrm{Na}^{*}$ approach each other. We speak of a bond stretching attraction mechanism. It is the primary cause of the kind of electronic to vibrational energy transfer observed, for in the next moment (Fig. 4c), a situation is reached in which the excited state of the system turns out to have a total energy which is nearly degenerate with that of the groundstate of the three heavy particles at the same geometry. This cannot be seen from Fig. 4 but it is one of the explicit results of the quantum chemical calculation that this crossing between the excited and ground state potential energy surfaces, at these low initial kinetic energies, is reached by stretching the $\mathrm{H}_{2}$ molecular bond distance as the $\mathrm{Na}^{*}$ approaches the molecule. This stretching is then the precursor of the vibrational excitation. In a geometry close to this crossing of the two potential energy surfaces, an electronic transition (electron jump) may occur with a high probability and the system finds itself suddenly in its electronic ground state (Fig. 4d). The ground state of the combined $\mathrm{NaH}_{2}$ system correlates, of course, with the 3 s ground state of the $\mathrm{Na}$ atom. Now the interaction between $\mathrm{Na}$ and $\mathrm{H}_{2}$ becomes strongly repulsive and the particles quickly recede on a trajectory which may overcompensate the initial attraction (Fig. 4e). At the same time the force between the two $\mathrm{H}$ atoms becomes attractive, the $\mathrm{H}_{2}$ finds itself in a non-equilibrium situation and the two $\mathrm{H}$ atoms move back towards their equilibrium distance. Thus a specific vibration of the molecule is excited, depending on the $\mathrm{H}$ $\mathrm{H}$ distance at the moment of the electron jump. One finds that the experimen-
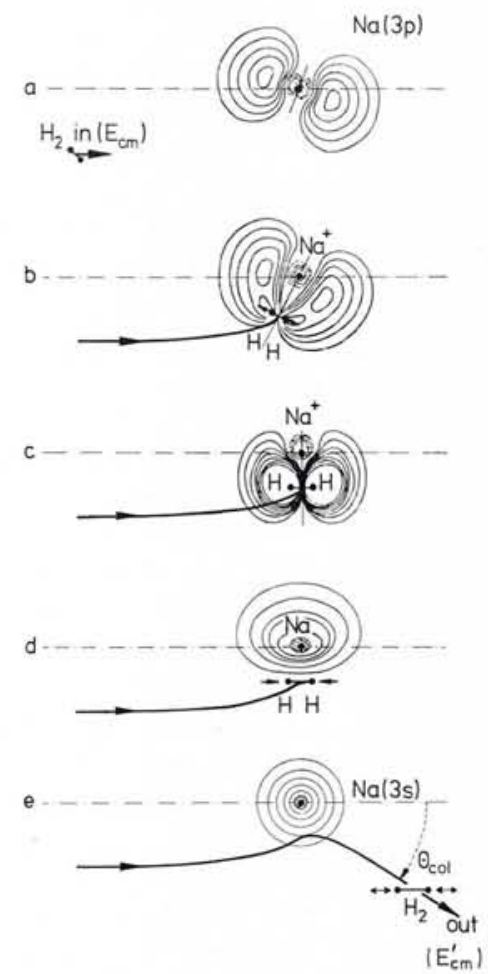

Fig. 4-Schematic picture of the dynamics in an individual electronic to vibrational energy transfer process between $\mathrm{Na}^{\circ}$ and $\mathrm{H}_{2}$. The $\mathrm{H}_{2}$ trajectory is shown (heavy full line) together with contour maps of the valence electron density at consecutive stages of the interaction. Note that the $\mathrm{H}_{2}$ molecular bond distance increases from approx. 1.7 to 2.2 a.u. from Fig. 4 a to $4 c$ as the calculations show. This corresponds to stretching the molecule so much that $v=2$ can be excited. 

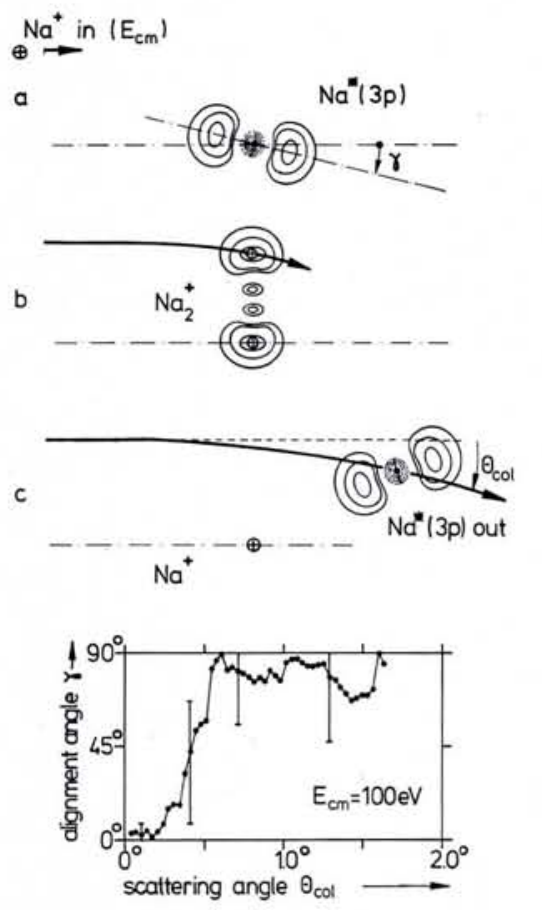

Fig. 5 - Schematic illustration of a charge transfer process (electron jump) in a collision of an excited $\mathrm{Na}(3 p)$ atom and a $\mathrm{Na}^{+}$ ion at three consecutive times $(5 a-c)$ during the interaction process, depicting the initial orbital alignment angle $\gamma$ of the 3p electron, the trajectory of the ion (heavy line) and its scattering angle $\theta_{\text {col }}$ after picking up the electron. The lower part shows the measured initial alignment angle $\gamma$ for which the charge transfer probability is a maximum as a function of the scattering angle $\theta_{\text {col }}$.

tally observed quantum numbers correspond very well to the simple predictions derived in this way from the potential surface calculations.

In order to develop this rather detailed but simple model picture a number of further results from the crossed molecular beam experiments had to be exploited which cannot be discussed here in detail. We simply mention that the angular distribution (scattering angle $\theta_{\text {col }}$ in Figs. 3 and 4 d) of the scattered products yields very important information for comparing theory and experiment and can be measured unambiguously in such a crossed beam experiment. Furthermore, in some favourable cases we may even go so far as to use the laser polarisation in the atomic excitation process to prepare the $\mathrm{Na}^{*}$ with well-defined orbital alignment of its valence electron, as was implied in Fig. 4a. Even such very detailed studies may be understood now on at least a semi-quantitative $a b$ initio basis ${ }^{7}$ ).

These kinds of polarisation studies, using lasers both to prepare atoms prior to a collision process and identify an atomic charge cloud distribution after a collision process, have become increa- singly popular during recent years. Using the polarisation of the exciting laser light it is possible to impose specific molecular symmetries prior to an interaction and to observe the specific reactivity of, say, a $\Sigma$ or a $\Pi$ molecular state as well as the different reactivities for different electronically excited states that the specific shape of the electron orbital creates. Such effects might well be expected from visualising photophysical elementary processes in the way depicted by Fig. 4: Just imagine the initial atomic orbital to be rotated by $90^{\circ}$ and it becomes difficult to see how the charge cloud might be transferred to the molecule. In fact, in the case of $\mathrm{Na}^{*}+$ $\mathrm{N}_{2}$ collisions, the alignment of the electron orbital as depicted in Fig. 4a has been found experimentally to be most favourable for the process, although by no means exclusive. For other processes such as inelastic $\mathrm{Na}^{+}+\mathrm{Na}^{*}$ collisions it is almost possible to switch the impact excitation on or off by changing the orbital alignment. And in reactive processes involving higher excited states, it was found that a Na- $4 d$ orbital was extremely effective in producing $\mathrm{NaO}$ products in reactions with $\mathrm{NO}_{2}$ and $\mathrm{O}_{2}$ while the 5 s orbital supplying almost the same energy led to no reaction at all ${ }^{8}$ ).

Such specificity in defining primary photophysical processes obviously opens a wide range of new types of study and, at the moment, we are only just beginning to exploit the possibilities. Charge transfer, one of the mechanisms of basic importance in the primary steps of many more complex photophysical processes might also be studied in this way. As a start one may investigate a simple model system where an atom excited by photon absorption interacts with an ion to which it transfers its valence electron while the two particles pass each other. As a convenient system we have studied

$$
\begin{aligned}
& \mathrm{Na}^{+} \text {(fast) }+\mathrm{Na}^{*} \text { (slow) } \rightarrow \\
& \mathrm{Na}^{*} \text { (fast) }+\mathrm{Na}^{+} \text {(slow) }
\end{aligned}
$$

The experimental set-up used is very similar to the one displayed in Fig. 3 except that the molecular beam is replaced by an ion beam and the fast neutral product atoms are detected. Again a laser is used to excite the atoms prior to collision and we may use the laser polarisation for defining the alignment angle of the electronic orbital with respect to the initial ion velocity prior to the transfer process. We may depict what happens during such an interaction, very schematically, as in Fig. 5. (The scheme is similar to Fig. 4 but not based on such detailed calculation and therefore should not be taken too literally.) The molecular beam experi- ment ${ }^{9}$ ) gives us information about the alignment angle $\gamma$ of the electron orbital which is most effective in allowing the electron jump to occur during the course of the collision. The result is shown in the lower part of Fig. 5. We see that at very small scattering angles $\theta_{\text {col }}<0.3^{\circ}$ (i.e. large impact parameters probing mainly the long range interaction leading to an electron jump over a large distance) the optimum charge transfer is achieved when the electron orbital is aligned nearly parallel to the incoming ion beam $\left(\gamma=0^{\circ}\right)$. In contrast, at somewhat larger scattering angles $\theta_{\text {col }}>$ $0.6^{\circ}$ (closer encounter of the collision partners), the best matching geometry seems to be for the electronic orbital to be aligned initially perpendicular to the ion beam $\left(\gamma=90^{\circ}\right)$.

Although this interesting experimental observation is, at the moment, not yet fully understood, we are very optimistic that a quantum mechanical interpretation will be possible in the near future, based on the known $\mathrm{Na}_{2}^{+}$potentials and coupling matrix elements. This, of course, is still very far from any of the complex photophysical processes of practical importance mentioned above. They normally occur in a very specific and structured environment comprising many large molecules. However, molecular beams also lead us one step further in the preparation of molecular aggregates. The newly flourishing field of cluster physics uses molecular beams very intensively to create objects ranging from just a few up to thousands of atoms or molecules ${ }^{10}$ ). Here I only wish to describe briefly an example from this new field which follows our line of thought further: What will happen if we excite a specific atom in a molecular compound which is preformed, rather than exciting one of them before the interacting particles collide?

Fig. 6 illustrates what we have found in a cluster consisting of one (or several) argon atoms and one benzene molecule, using a molecular beam cluster source and a synchrotron storage ring light source ${ }^{11}$ ).

By exciting this complex with photons of wavelengths tuned across the region of the first $4 s^{3} p^{-1}$ and $4 s^{\prime} 3 p^{-1}$ resonance lines (abbreviated notation) of the free argon atom, we can show that it is indeed possible specifically to excite the argon atom to these states, thereby creating a $3 p$-electron hole. What we then observe are two possible exit channels; either the $\mathrm{Ar} \cdot$ benzene $^{+}$complex ion is detected or a fragment, i.e. a benzene $^{+}$ion is seen. In both cases, however, we observe a pronounced 
structure in the spectra obviously related to the argon resonance lines. If the complex is detected, we see mainly a red shift, while if the fragment is detected, the line is broad and blue shifted. Although unable to understand these observations in detail, we are nevertheless led to speculate about the mechanism, based on our previous discussion of charge transfer in collisions: The 3-p hole created in the argon atom may subsequently be transferred to the benzene molecule, whereby enough energy is gained to eject the outer $4 \mathrm{~s}$ argon electron since the total energy of the system is larger than necessary for ionising the molecule. Thus we observe the ionic cluster and its fragments. We have named this process intramolecular Penning ionisation (IPI). This kind of specific localised excitation might well be exploited in future studies on increasingly larger molecular aggregates.

It is hoped that these few examples have illustrated some of the many new facets of molecular beam studies by which very detailed information on primary photophysical and photochemical processes may be obtained in cold molecules and molecular clusters. The use of lasers and synchrotron radiation is instrumental in these studies as are complex theoretical calculations which will

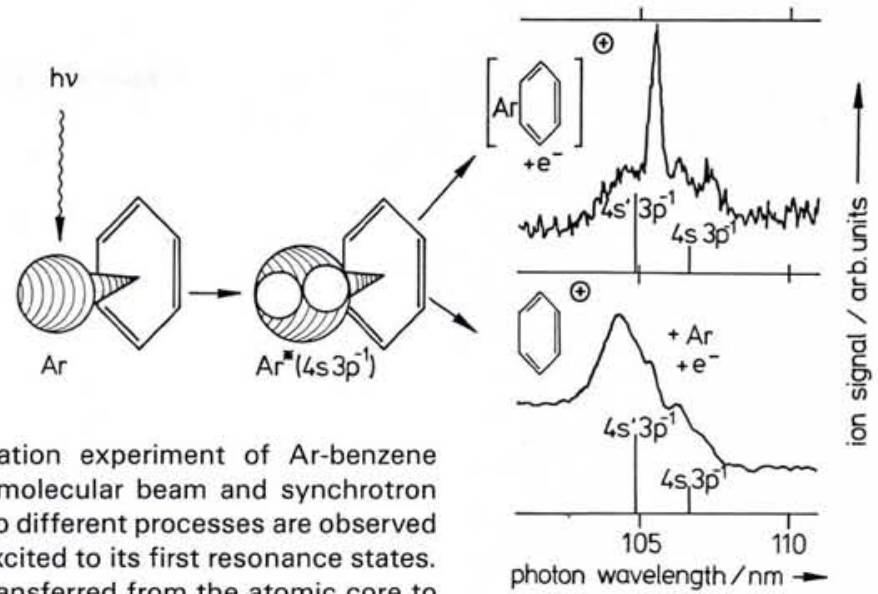

Fig. 6 - Photoionisation experiment of Ar-benzene clusters using a cold molecular beam and synchrotron radiation. Note that two different processes are observed as the argon atom is excited to its first resonance states The $3 p$-hole may be transferred from the atomic core to the benzene molecule thus leading to ionisation of the cluster (upper spectrum) or to ionisation and fragmentation (lower spectrum). Shown are the respective ion yield as a function of the photon wavelength and the positions of the resonance lines of free argon atoms.

eventually allow us to develop simple model pictures.

\section{REFERENCES}

1. Donovan R.J., Prog. React. Kinet. 10 (1979) 253

2. See in particular the whole issue: Euro phys. News 16 (1985) 1-16.

3. Wood R.W., Verh. d. deutsch. Phys. Ges. 13 (1911) 72.

4. Reiland W., Tittes U. and Hertel I.V., Phys. Rev. Lett. 48 (1982) 1389

5. Botschwina P., Meyer W., Hertel I.V. and Reiland W., J. Chem. Phys. 75 (1981) 5438. 6. Papierowska-Kaminski D., Persico M. and
Bonacic-Koutecky V., Chem. Phys. Lett. 113 (1985) 264.

7. Poppe D., Z. Phys. D: Atoms, Molecules and Clusters 1 (1986) 207.

8. Schmidt H., Weiss P.S., Mestdagh J.M., Covinsky M.H. and Lee Y.T., Chem. Phys. Lett. 118 (1985) 539.

9. Witte R., Campbell E., Richter Ch., Schmidt H. and Hertel I.V., Z. Phys. D 4 (1986) to be published.

10. See for instance a collection of papers on metal clusters edited by zu Putlitz G. and Träger F., in Z. Phys. D 3 (1986) in press.

11. Kamke W., Kamke B., Kiefl H.U. and Hertel I.V., Chem. Phys. Lett. 122 (1986) 356.

\section{NI KMEF}

The National Institute for Nuclear Physics and High Energy Physics invites

\section{NUCLEAR PHYSICISTS (PH-D)}

to apply for post-doctoral positions in its Nuclear Physics section. The Institute is involved in fundamental research of the structure of nuclei and nucleons with its $500 \mathrm{MeV}$ linear electron accelerator. The main research lines are elastic and inelastic electron scattering with large spectrometers enabling high-resolution single-arm as well as double-arm (coincidence) experiments. Part of the research program involves developing the capability to carry out intermediate energy nuclear physics research with $100 \%$ duty factor beams.

The ongoing program is carried out by a strong in-house staff (under direction of prof. dr. C. de Vries) in close collaboration with visiting teams from many laboratories outside the Netherlands.

The Ph-D applicants are expected:

- to have experience with intermediate energy nuclear physics;

- to have the potential to play a stimulating role in one of the groups involved with the on-going program.

The positions are available for a period of two years under contract with the Organisation for Fundamental Research of Matter (FOM).

Further information can be obtained from the Scientific Director of NIKHEF K, prof. dr. G. van Middelkoop, (tel. 020-5922162) and from prof. dr. C. de Vries (020-5922142).

Written applications, directed to the Scientific Director NIKHEF, Postbox 4396, 1009 AJ Amsterdam, including a curriculum vitae, list of publications and some references to be sent within one month after appearance of this advertisement. 